\title{
The Application of Improved Duncan-Chang Model in Unloading Soil
}

\author{
Yi He ${ }^{1,2}$ and Xuejun Chen ${ }^{1,3, *}$
}

${ }^{1}$ Faculty of Engineering, China University of Geosciences (Wuhan), Wuhan 430074, China; ${ }^{2}$ Civil Engineering Department, Luoyang Institute of Science and Technology, Luoyang 471023, China; ${ }^{3}$ Civil Engineering Department, Guilin University of Technology, Guilin 541004, China

\begin{abstract}
On the basis of the representative samples of silty clay found in Wuhan, China, the lateral unloading of soil's stress path produced by excavating foundation pit engineering, was simulated by triaxial experiment. A series of consolidated-drained true triaxial test and normal triaxial test were conducted. According to the results of tests, the parameter of the Duncan-Chang Model was determined. A modulus formula was used for the foundation soil in the lateral unloading stress path tests to replace the modulus formula of Duncan-Chang Model based on the $\sigma_{3}=$ const . Moreover, the Duncan-Chang hyperbola nonlinear elastic constitutive model was used to simulate the plane strain test. A method to improve the ability of Duncan-Chang model in order to take into account the effects of the intermediate principal stress on the strength and deformation was presented as well as all the model parameters were also determined. The adaptability of the model for unloading the stress path was verified by comparing the theoretical stress-strain relationship and empirical stress-strain relationship.
\end{abstract}

Keywords: Foundation pit excavation, lateral unload, improved Duncan-Chang model.

\section{INTRODUCTION}

In excavation engineering, when soil is in the state of unloading, it experiences upheaval in the bottom of the excavation pit. Problems in the lateral displacement of soil induced by unloading and general problem in the geotechnical loading are noticeably different. Numerical analysis was carried out on the foundation pit excavation with the conventional earth applied by loading test parameter and the design had various I evitable errors.

In this paper, excavation process of actual pit was simulated. A series of consolidated-drained true triaxial test and normal triaxial test were conducted. According to the experimental results, under the condition of drainage of foundation pit in lateral load shedding, the constitutive model of soil was applied after the excavation of foundation pit. Instead of Duncan-Chang model the deformation modulus for lateral unloading was used for the calculation [1] of tangent modulus formula $[2,3]$. The Duncan-Chang nonlinear elastic model was applied to the plane strain condition, in view of the fact that the Duncan-Chang model does not reflect the principal stress, therefore, the Duncan Chang model was improved. . In addition, the parameters of the model were determined, and the income was observed by the income relationship curves of stress-strain and testing curves of stress - strain through the comparative analysis with theoretical calculation, in order to validate the adaptability of the model for unloading engineering of this special stress path.

\footnotetext{
*Address correspondence to this author at the 12 Jiangan Road Guilin, Guilin University of Technology, Guilin 541004, China;

Tel: +8613907738446; E-mail: chenxj@glut.edu.cn
}

\section{EXPERIMENTAL STUDY ON CONSTITUTIVE MODEL UNDER THE CONDITION OF DRAINAGE OF UNLOADING SOIL}

\subsection{Two Dimensional Stress-Strain Test}

This test mainly analysed the soil properties of the surrounding active area of the foundation pit, and simplified the force state approximation for unchanged vertical load in lateral unloading. Undisturbed soil samples were taken from Wuhan Shahu Power District high-rise residential building of foundation pit construction site after testing. The city of Wuhan is representative of the Yangtze River I terrace alluvial silty clay. The physical and mechanical indexes are shown in Table $\mathbf{1 .}$

Table 1. Physical and mechanical parameters of soil samples.

\begin{tabular}{|c|c|c|c|c|c|}
\hline$\omega(\%)$ & $\gamma\left(\mathbf{g} / \mathbf{c m}^{3}\right)$ & $c(\mathbf{k p a})$ & $\varphi\left({ }^{\circ}\right)$ & $\boldsymbol{e}$ & $\boldsymbol{I}_{p}$ \\
\hline \hline 33.4 & 1.9 & 21.6 & 22.7 & 0.97 & 13.0 \\
\hline
\end{tabular}

The sample was saturated, respectively by $50,100,150$, $200,250,300 \mathrm{KPa}$ confining pressure of isotropic consolidation, maintained after consolidation, and was immediately sheared with a constant vertical pressure control. In the process of shearing, and classification of unloading, each stage of unloading grade had $10 \mathrm{KPa}$ with stress as the control variable. The stress, and displacement, in each load case were measured in the whole test process suggesting $\sigma_{1}>\sigma_{2}=\sigma_{3}$. Results of the stress-strain curve are shown in Fig. (1). 


\subsection{Triaxial Loading Stress-Strain Test}

Three dimensional test for testing soil stress state was carried out on the ZSY - 1 type by true triaxial test apparatus, with samples of saturation, and with the consolidation of normal three axial test confining the pressure of isotropic consolidation, maintained after consolidation, was immediately sheared. $\sigma_{1}$ and $\sigma_{2}$ remained unchanged due to vertical deformation of pressure control in the process of shearing (namely: $\left.\varepsilon_{2}=0\right)$ ), $\sigma_{3}$ is grade unloading, each stage of unloading had $10 \mathrm{KPa}$, with stress as the control variable. The stress and displacement were measured in each load case, in the whole testing process $\sigma_{1}>\sigma_{2}>\sigma_{3}$.

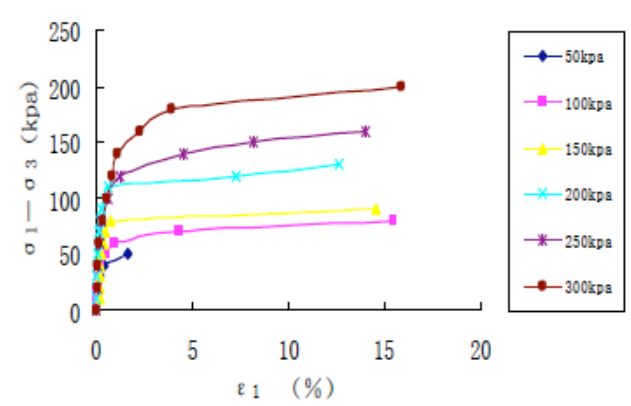

Fig. (1). The stress-strain curves for drained test of normal triaxial.

According to the drainage of lateral plane, strain conditions were determined by using true triaxial instrument under the unloading test to obtain the stress-strain relation curve as shown in Fig. (2).

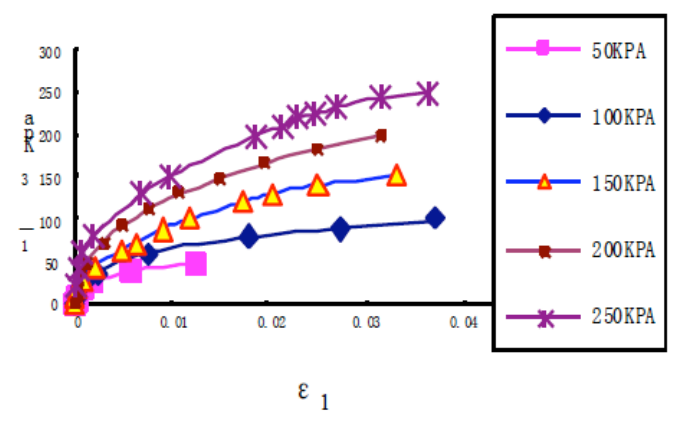

Fig. (2). The stress-strain curves for drained test of true triaxial.

\subsection{Experimental Study on Constitutive Model of Un- loading Soil}

At present, among the nonlinear elastic models used in engineering applications, the most significant one is the Duncan-Chang $[2,3]$ model. The soil has complicated deformation features, such as dilatancy, anisotropy, and the influence of stress path. In some soil nonlinear elastic or elastic-plastic model, for general loading conditions, the satisfactory results can be obtained, but for the special loading condition, the results will not be reasonable. In addition, the model is based on the failure criterion of Mohr-colin in which, $\sigma_{2}=\sigma_{3}$, without considering the middle stress of $\sigma_{2}$. In the original Duncan Zhang model verification application, - there are certain inevitable errors in the deformation test of soil under plane strain condition. Therefore, in order to make the model simulate the actual soil engineering properties, some improvements must be made.
By unloading soil deformation modulus formula [1] side of foundation pit excavation.

\subsubsection{Analysis of Duncan-Chang Model Parameters}

Li Shoude, Zhang Tuqiao, et al. through the lateral unloading stress path test analysed the deformation laws of the soil behind the wall lateral natural foundation consolidation in foundation pit excavation unloading process, based on the hyperbolic hypothesis, and cited the calculation parameters of Duncan-Chang model for deducing deformation modulus formula for natural soil excavation in foundation pit lateral unloading process, which is represented as follows:

$$
\begin{aligned}
& E=E_{i}\left(1-R_{f} \frac{(1-\sin \varphi)\left(1-k_{0}\right) \sigma_{1}}{2 c \cos \varphi+2 k_{0} \sigma_{1} \sin \varphi}\right)^{2} \times \\
& {\left[1-R_{f 1} \frac{(1+\sin \varphi)\left(k_{0} \sigma_{1}-\sigma_{3}\right)}{2 c \cos \varphi+\left[2 \sin \varphi-(1+\sin \varphi)\left(1-k_{0}\right)\right] \sigma_{1}}\right]^{2}}
\end{aligned}
$$

In the formula given below, $E_{i}$ is a function of $K_{0}$ consolidation state of the $\sigma_{1}$ :

$$
E_{i}=k p_{a}\left(\frac{\sigma_{3}}{p_{a}}\right)^{n}
$$

Using the true triaxial and normal triaxial, in isotropic consolidation, i.e. $\sigma_{1}=\sigma_{2}=\sigma_{3}$, therefore $K_{0}=1$, then (1) type into:

$$
\lg \frac{\sigma_{3}}{p_{a}} E=E_{i}\left[1-R_{f 1} \frac{(1+\sin \varphi)\left(\sigma_{1}-\sigma_{3}\right)}{2 c \cos \varphi+2 \sigma_{1} \sin \varphi}\right]^{2}
$$

In addition, while testing the lateral unloading stress path , deformation of the volume curve in the test was more difficult to ascertain the practical expression of the Poisson ratio $\mu$. But tests showed $\varphi$ that the lateral unloading stress path varied to a lesser extent [4].

By formulas (2) and (3), it can be observed that, there were seven parameters in the Duncan-Chang model, i.e.: $k, R_{f}, k_{b}, m, n, c$, and $\varphi$.

\subsubsection{To Determine the Parameters of the Duncan-Chang Model [5, 6]}

1) $C, \varphi$ value determination

According to the data of normal triaxial test results, obtained under different confining pressure and different principal curves of stress and axial strain were obtained rendering the molar under different confining pressures and stress circle, as shown below:

$c=16.77 \mathrm{kpa}, \varphi=22.3^{0}$,

2) $E_{i}, R_{f}$ value determination 
Table 2. $E_{i}$ and $R_{f}$ parameters of $\lg \left(\sigma_{3} / p_{a}\right)$ silty clay.

\begin{tabular}{|c|c|c|c|c|c|c|c|}
\hline $\boldsymbol{\sigma}_{\mathbf{3}}(\mathbf{k p a})$ & $\left(\boldsymbol{\sigma}_{\mathbf{1}}-\boldsymbol{\sigma}_{\mathbf{3}}\right)_{\mathbf{f}}(\mathbf{k p a})$ & $\mathbf{a}$ & $\boldsymbol{E}_{\boldsymbol{i}}(\mathbf{k p a})$ & $\mathbf{b}$ & $\left(\boldsymbol{\sigma}_{\mathbf{1}}-\boldsymbol{\sigma}_{\mathbf{3}}\right)_{\mathrm{ult}}(\mathbf{k p a})$ & $\boldsymbol{R}_{\boldsymbol{f}}(\mathbf{k p a})$ & Average \\
\hline \hline 50 & 0 & $3.08 \mathrm{E}-05$ & 32457.35 & 0.018044 & 55.42 & 0.902219 & 0.990803 \\
\hline 100 & 20 & $3.24 \mathrm{E}-05$ & 30835.27 & 0.012385 & 80.74 & 0.987647 & 0.96 \\
\hline 250 & 160 & $2.54 \mathrm{E}-05$ & 39416.50 & 0.006173 & 161.99 & 0.971314 & \\
\hline 300 & 200 & $2.47 \mathrm{E}-05$ & 40467.75 & 0.004857 & 205.88 & \\
\hline
\end{tabular}

Table 3. $\lg \left(\sigma_{3} / p_{a}\right)$ and $\lg \left(E_{i} / p_{a}\right)$ parameters of silty clay in different cell pressure.

\begin{tabular}{|c|c|c|c|c|c|}
\hline$\sigma_{3}(\mathbf{k p a})$ & $\boldsymbol{\sigma}_{3} / \boldsymbol{p}_{\boldsymbol{a}}$ & $\lg \left(\boldsymbol{\sigma}_{3} / \boldsymbol{p}_{\boldsymbol{a}}\right)$ & $\boldsymbol{E}_{\boldsymbol{i}}(\mathbf{k p a})$ & $\boldsymbol{E}_{\boldsymbol{i}} / \boldsymbol{p}_{\boldsymbol{a}}$ & $\lg \left(\boldsymbol{E}_{\boldsymbol{i}} / \boldsymbol{p}_{\boldsymbol{a}}\right)$ \\
\hline \hline 50 & 0.5 & 2.511215 & 32457.35 & 324.5 & -0.301 \\
\hline 100 & 1 & 2.488974 & 30835.27 & 308.3 & 0.000 \\
\hline 250 & 2.5 & 2.595496 & 39416.50 & 394.0 & 0.398 \\
\hline 300 & 3 & 2.607026 & 40467.75 & 404.6 & 0.477 \\
\hline
\end{tabular}

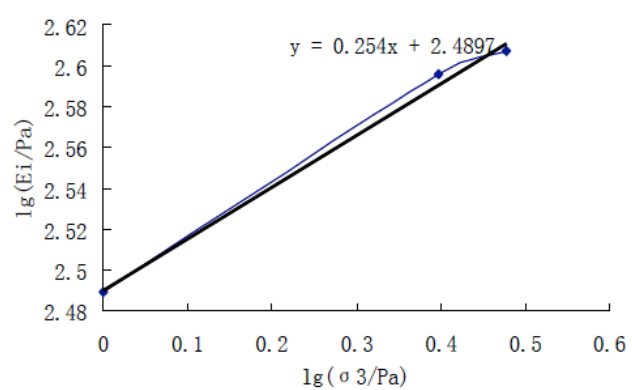

Fig. (3). The relationship between $\lg \left(\sigma_{3} / p_{a}\right)$ and $\lg \left(E_{i} / p_{a}\right)$.

Because the actual stress-strain relationship was in line with the basic assumptions of the hyperbola, the stress-strain hyperbolic $\left(\sigma_{1}-\sigma_{3}\right)-\varepsilon_{1}$ was converted into a linear relationship between $\varepsilon_{1} /\left(\sigma_{1}-\sigma_{3}\right)-\varepsilon_{1}$ and different confining pressures are represented as intercepts $a$ and $b$ whose values are shown in Table 2.

3) $K, n$ value determination

Take the atmospheric pressure $P_{a}=101.3 \mathrm{Kpa}$,

$\lg \left(E_{i} / p_{a}\right)$ was obtained.

The values of confining pressure, are shown in Table $\mathbf{3}$, and the

$\lg \left(\sigma_{3} / p_{a}\right): \lg \left(E_{i} / p_{a}\right)$

linear relation mapping, is shown in Fig. (3), which can be used to obtain the parameters $\mathrm{K}$, and $\mathrm{n}$ value which were, $\mathrm{K}=308.8$, and $\mathrm{n}=0.254$.

4) $K_{b}, m$ value determination

Take the atmospheric pressure $\mathrm{Pa}=101.3 \mathrm{Kpa}$, $B=\left(\sigma_{1}-\sigma_{3}\right) / 3 \varepsilon_{V}, \lg \left(B / P_{a}\right)$ was obtained andB values of

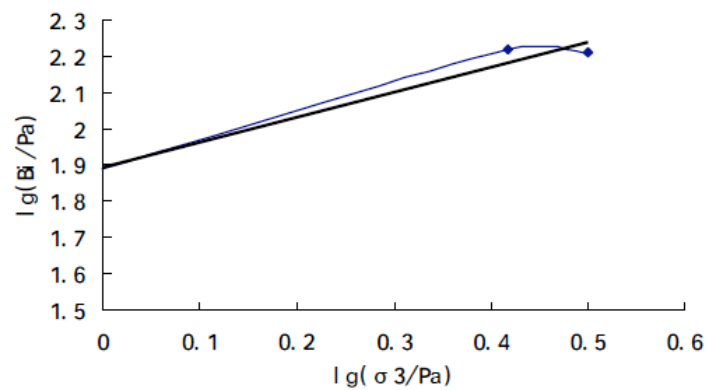

Fig. (4). The relationship between $\lg \left(\sigma_{3} / p_{a}\right)$ and $\lg \left(B / p_{a}\right)$.

confining pressure, are shown in Table 4. The $\lg \left(\sigma_{3} / p_{a}\right) \sim$ $\lg \left(B / p_{a}\right)$ linear relation mapping is shown in Fig. (4), which can be used to obtain the parameters $K_{b}$, and $\mathrm{m}$, and these were, $K_{b}=66.24$, and $\mathrm{m}=0.764$.

Thus, the parameters of Duncan-Chang model were obtained by the experiment of soil, listed in Table 5 .

5) Duncan-Chang hyperbola model for plane problems

Duncan Chang hyperbolic E-K model parameter was obtained by the normal triaxial test to determine the deformation calculation with three ordinary specimen with its natural. Compared with the measured results, the calculation of deformation of plane strain $\mathrm{s} K_{b}$ and specimen parameters today with three axis test for determining, for $\sigma_{3}$, the simulation of excavation process will the deviatoric stress is divided into several components, each increment in mean stress on the hyperbolic model for $E_{i}$ and, the formation of flexibility matrix

$[c]=[D]^{-1}$ 
Table 4. $\lg \left(\sigma_{3} / p_{a}\right)$ and $\lg \left(B / p_{a}\right)$ parameters.

\begin{tabular}{|c|c|c|c|c|c|c|c|}
\hline$\sigma_{3}(\mathrm{kpa})$ & $\lg \left(\sigma_{3} / p_{a}\right)$ & $\left(\sigma_{1}-\sigma_{3}\right) f(\mathbf{k p a})$ & $\left(\sigma_{1}-\sigma_{3}\right)(\mathbf{k p a})$ & $\varepsilon_{v}(\%)$ & $B(\mathbf{k p a})$ & $B / p_{a}$ & $\lg \left(B / p_{a}\right)$ \\
\hline \hline 50 & -0.301 & 50 & 35 & 0.0070 & 3333.33 & 33.33 & 1.522 \\
\hline 100 & 0.000 & 80 & 56 & 0.0048 & 7777.77 & 77.78 & 1.891 \\
\hline 250 & 0.398 & 160 & 112 & 0.0055 & 13575.76 & 135.76 & 2.132 \\
\hline 300 & 0.477 & 200 & 140 & 0.0011 & 84848.48 & 848.48 & 2.929 \\
\hline
\end{tabular}

Table 5. Parameters of Duncan-Chang model.

\begin{tabular}{|c|c|c|c|c|c|c|}
\hline $\boldsymbol{C}(\mathbf{k p a})$ & $\boldsymbol{\varphi}$ & $\boldsymbol{R}_{\boldsymbol{f}}$ & $\boldsymbol{K}$ & $\boldsymbol{n}$ & $\boldsymbol{K}_{\boldsymbol{b}}$ & $\boldsymbol{m}$ \\
\hline \hline 16.77 & 22.3 & 0.96 & 308.8 & 0.254 & 66.24 \\
\hline
\end{tabular}
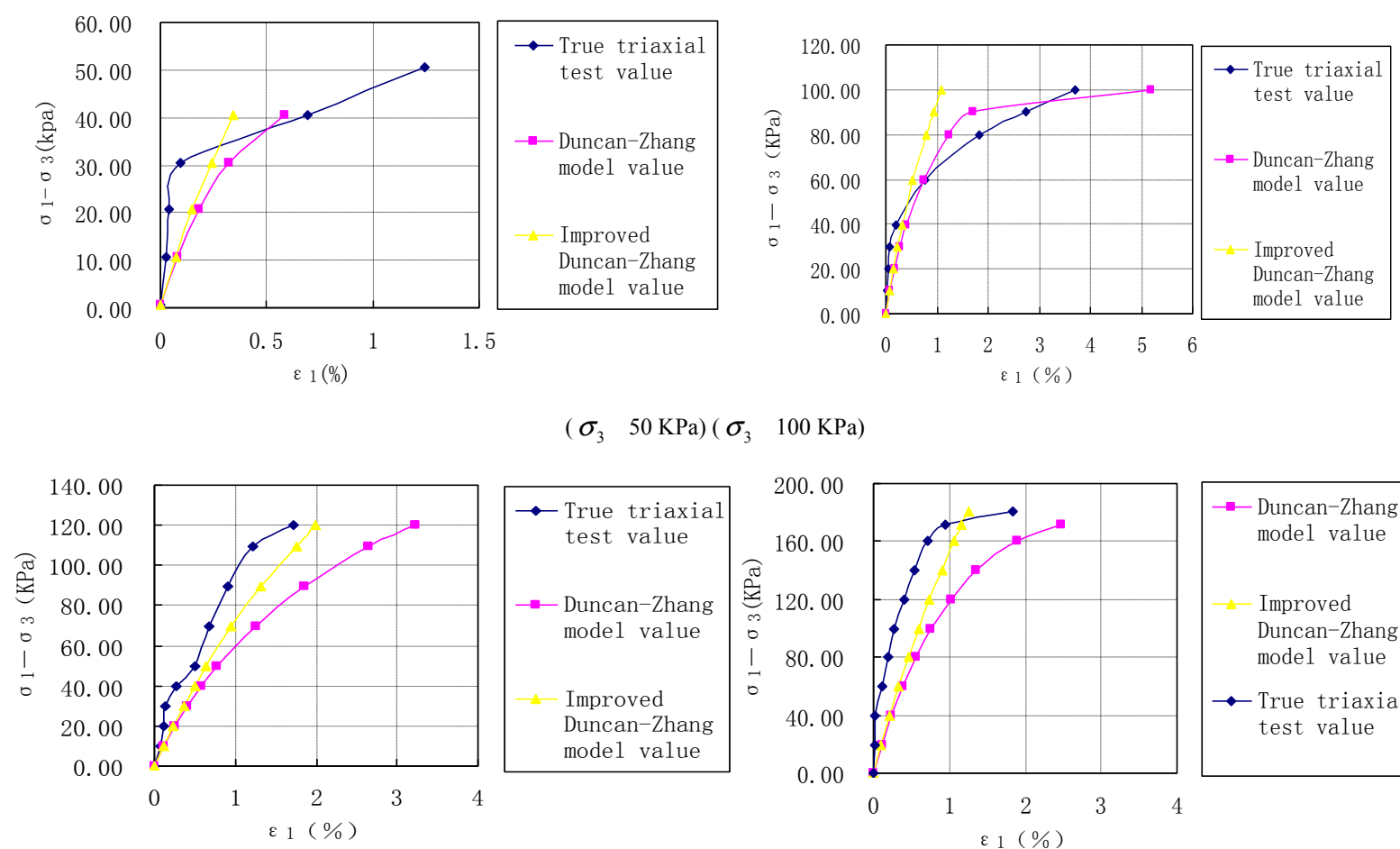

$\left(\begin{array}{ll}\sigma_{3} & 50 \mathrm{KPa}\end{array}\right)\left(\begin{array}{ll}\sigma_{3} & 100 \mathrm{KPa}\end{array}\right)$
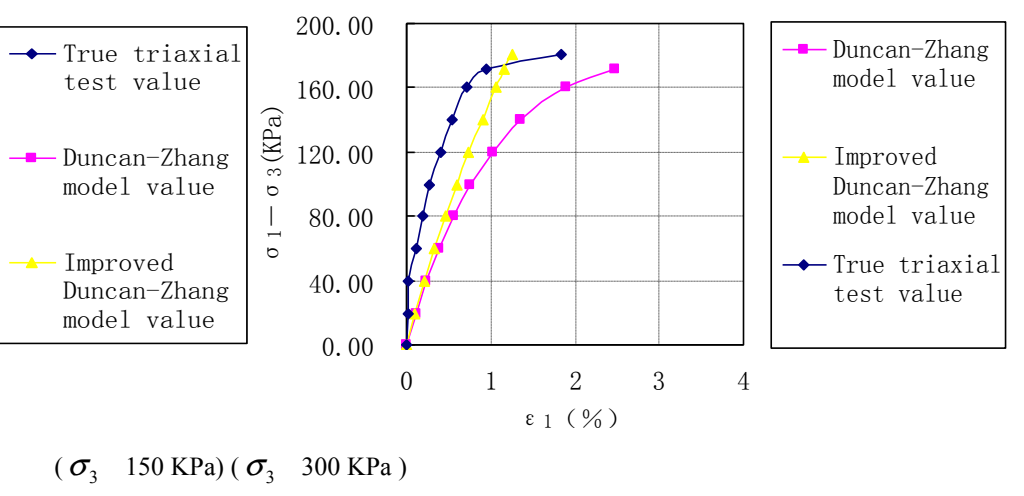

Fig. (5). The curves for $\left(\sigma_{1}-\sigma_{3}\right) \sim \varepsilon_{1}$.

wherein:

$$
[D]=\frac{3 K}{9 K-E}\left[\begin{array}{ccc}
3 K+E & 3 K-E & 0 \\
3 K-E & 3 K+E & 0 \\
0 & 0 & E
\end{array}\right]
$$

According to the different load of $\Delta \varepsilon_{1}$ accumulation, the corresponding stress-strain curves were drawn. The curve and the test curve of Duncan Chang model income theory really three shaft under plane strain conditions as shown in Fig. (5).

\section{The main effect of stress $\sigma_{2}$ consideration}

The Duncan-Chang model does not consider the intermediate principal stress on strength and deformation effect of the $\sigma_{2}$ resulting in low deformation modulus of soil in the specific calculation process of large deformation of soil. Therefore, many domestic and foreign scholars have put forward various methods for the application of the model and at the same time, have tried to improve the model, by overcoming its weaknesses. At present, the modified elastic modulus, Poisson's ratio and modified Mohr-Colin's method are the two kinds of methods [6-8] that have been proposed, 
Table 6. Parameters of improved Duncan-Chang model.

\begin{tabular}{|c|c|c|c|c|c|c|}
\hline $\boldsymbol{C}($ kpa $)$ & $\boldsymbol{\varphi}$ & $\boldsymbol{R}_{\boldsymbol{f}}$ & $\boldsymbol{K}$ & $\boldsymbol{n}$ & $\boldsymbol{K}_{\boldsymbol{b}}$ & $\boldsymbol{m}$ \\
\hline \hline 16.77 & 22.3 & 0.96 & 308.8 & 0.254 & 66.24 & 0.764 \\
\hline
\end{tabular}

but the study used only one correction method of elastic modulus for the simulation of plane strain test results, which reflects the complex degree of stress-strain characteristics to verify the improved model. Thus, it provides the reference for the selection of numerical analysis in geotechnical engineering constitutive model.

In order to consider the influence of intermediate principal stress of $\sigma_{2}$, the original Duncan model of confining pressure $\sigma_{3},\left(\sigma_{2}+\sigma_{3}\right) / 2$ was used instead of partial stress $\left(\sigma_{1}-\sigma_{3}\right), \sigma_{1}-\left(\sigma_{2}+\sigma_{3}\right) / 2$ to keep the Mohr-colin's standards unchanged, and to consider the degree of influence of intermediate principal stress $\sigma_{2}$ on stress-strain characteristics of stress paths.

The improved model for considering the influence of intermediate principal stress $\sigma_{2}$ changed the corresponding parameter and the expression of tangent modulus of elasticity model corresponding to:

$$
E=E_{i}\left[1-R_{f 1} \frac{(1+\sin \varphi)\left(\sigma_{1}-\frac{\sigma_{2}+\sigma_{3}}{2}\right)}{2 c \cos \varphi+2 \sigma_{1} \sin \varphi}\right]^{2}
$$

1) The parameters of the improved model

The improved Duncan Chang model parameters still include seven original parameters of Duncan Chang nonlinear model, namely $\mathrm{K}, K_{b}, \mathrm{~m}, \mathrm{n}, R_{f}$, c and $\varphi$. The improved model parameters can still be obtained from the normal triaxial test. The nonlinear elastic model with improved parameter values is shown in Table 6.

2) Duncan-Chang modified hyperbola model for plane problems

The Duncan-Chang hyperbola model improved the method which is still used for verification before unloading soil in plane strain side of the foundation pit excavation. The obtained experimental calculation curves and the original Duncan Chang model calculation curves were drawn together, as shown in Fig. (5).

\section{CONCLUSION}

By comparing the graph, it was observed that the calculated value and experimental value of Duncan-Zhang model were larger, even with the modified Duncan-Chang model. However, the calculated value, experimental value and anastomosis were not appropriate. Following are the reasons for this difference:

1) Duncan Chang nonlinear elastic model was established based on the conventional 2D model of normal triaxial test, and model parameters were obtained by the ordinary normal triaxial test. Although the value of this parameter method is simple and convenient, its application to $3 \mathrm{D}$ in plane strain test needs to be verified effective calculation results .

2) Duncan Chang nonlinear elastic model was set up based on the normal triaxial loading test and constant confining pressure conditions. Therefore, it is suitable for general loading conditions, but for the special loading condition, reasonable results are not obtained. In the excavation of foundation pit engineering, function of soil load mode is different from the general loading, therefore, using the model to study the deformation of soil under the condition of plane strain loading unloading is unreasonable.

$3)$ In the tests of the plane strain determining damage deviatoric stress ratio of $\left(\sigma_{1}-\sigma_{3}\right)$ three axial test was effective, and the failure strain with three triaxial test was slightly low . According to the Mohr-Colin failure criterion, plane strain test of $\varphi$ angle was high than three triaxial test, whereas, the three axis measured $\varphi$ angle for plane strain problem was low. This is because the main stress on the strength of the Mohr-Colin and the generated error were not considered. The reason behind failure in the application of hyperbolic model and the problem in plane strain is faulty selection, thereby causing difference in the calculation curve and experimental curve of income.

4) In view of the influence of middle principal stress determined by Duncan Chang model, the improvement in Duncan Chang model was made to consider the influence of intermediate principal stress on the strength of soil, the calculated curve was derived from it can be seen that the Duncan-Chang model curve after improvement is located in the former Duncan-Chang model, i.e. tangent of the elastic model curve after correction in the calculated curve was higher than the original Duncan-Chang model results.

This also makes the calculation more close to the curve obtained by the plane strain test. Although the improved model improved the tangent modulus curve, on the contrary, it can be observed from the graph that the strain calculation prediction is still smaller than the experimental value, which suggests that the Duncan-Chang model has limited capacity for determining the stress strain properties of complex stress state which needs to be further improved.

\section{CONFLICT OF INTEREST}

The authors confirm that this article content has no conflict of interest.

\section{ACKNOWLEDGEMENTS}

Declared none.

\section{REFERENCES}

[1] S. D. Li, T. Q. Zhang, B. T. Wang, and Z. F. Lu, "Modulous formula for foundation soil under lateral unloading during excavation", Civil Engineering Journal, vol. 35, no. 5, pp. 70-74, 2002. 
[2] J. M. Duncan and C. Y. Chang, "Nonlinear analysis of stress and strain in soils", Journal of the Soil Mechanics and Foundation Division, vol. 96, no. 5, pp. 1629-1653, 1970.

[3] P. V. Lade, "Elasto-plastic Stress-Strain Theory for Cohesionless Soil with Curved Yield Surfaces", Soilds Structures, vol. 13, pp. 1019-1035, 1977.

[4] J. H. Qian, The principle and calculation of earthwork, Beijing: Chinese water conservancy and Hydropower Press, 2000.
[5] G. X. Li, Advanced soil mechanics, Beijing: Tsinghua University press, 2004.

[6] T. H. Lu and Z. D. Liu, Advanced soil mechanics, Beijing: Machinery Industry Press, 2006.

[7] Z. L. Liu, "Evaluation on developing level of unban agglomeration derived from resources exploration", Journal of Applied Sciences, vol. 13, no. 21, pp. 4702-4707, 2013

[8] X. Y. Zhang and S. W. Yan, Geotechnical plastic mechanics Foundation, Tianjin: Tianjin University press, 2004.

Received: July 09, 2014

Revised: October 12, 2014

Accepted: November 1, 2014

(c) He and Chen; Licensee Bentham Open.

This is an open access article licensed under the terms of the (https://creativecommons.org/licenses/by/4.0/legalcode), which permits unrestricted, noncommercial use, distribution and reproduction in any medium, provided the work is properly cited. 